\title{
Spaced Learning Enhances Subsequent Recognition Memory by Reducing Neural Repetition Suppression
}

\author{
Gui Xue ${ }^{1,2}$, Leilei Mei ${ }^{1,3}$, Chuansheng Chen ${ }^{3}$, Zhong-Lin Lu $^{2}$, \\ Russell Poldrack ${ }^{4}$, and Qi Dong ${ }^{1}$
}

\begin{abstract}
Spaced learning usually leads to better recognition memory as compared with massed learning, yet the underlying neural mechanisms remain elusive. One open question is whether the spacing effect is achieved by reducing neural repetition suppression. In this fMRI study, participants were scanned while intentionally memorizing 120 novel faces, half under the massed learning condition (i.e., four consecutive repetitions with jittered interstimulus interval) and the other half under the spaced learning condition (i.e., the four repetitions were interleaved). Recognition memory tests afterward revealed a significant spacing effect: Participants recognized more
\end{abstract}

\section{INTRODUCTION}

One ubiquitous phenomenon in learning and memory is the spacing effect. First demonstrated by Ebbinghaus $(1885 / 1964)$, the spacing effect refers to the fact that memory is better for items whose repetitions are interleaved (i.e., spaced learning [SL], also called distributed practice) than for those whose repetitions are massed (i.e., repetitions are adjacent in time massed learning $[\mathrm{ML}]$ ). Memory benefits from SL have been observed for various learning materials/tasks (e.g., verbal, nonverbal, and motor skills) and memory tasks (e.g., free recall, recognition, and skill performance) (for reviews, see Cepeda, Pashler, Vul, Wixted, \& Rohrer, 2006; Donovan \& Radosevich, 1999; Arthur, Bennett, Stanush, \& McNelly, 1998).

Many theoretical models have been proposed to account for the spacing effect, with varying explanatory power for different study materials and different memory tasks (Greene, 1989). Of particular interest to the present study is the short-term perceptual priming hypothesis (Russo, Parkin, Taylor, \& Wilks, 1998), which suggests that the spacing effect is a result of reduced perceptual priming during learning. This model is echoed in the neural model proposed by Wagner, Maril, and Schacter (2000), which suggests that repetition priming, characterized neurophysiologically as a decreased response after stimulus repetition, hinders subsequent memory (SM).

\footnotetext{
${ }^{1}$ Beijing Normal University, ${ }^{2}$ University of Southern California, ${ }^{3}$ University of California, Irvine, ${ }^{4}$ University of Texas, Austin
}

items learned under the spaced learning condition than under the massed learning condition. Successful face memory encoding was associated with stronger activation in the bilateral fusiform gyrus, which showed a significant repetition suppression effect modulated by subsequent memory status and spaced learning. Specifically, remembered faces showed smaller repetition suppression than forgotten faces under both learning conditions, and spaced learning significantly reduced repetition suppression. These results suggest that spaced learning enhances recognition memory by reducing neural repetition suppression.

Using recognition tasks, several studies have found strong spacing effects using nonverbal materials such as nonsense shapes (Cornoldi \& Longoni, 1977), unfamiliar faces (Mammarella, Russo, \& Avons, 2002; Russo et al., 1998), and nonwords (Mammarella, Avons, \& Russo, 2004; Mammarella et al., 2002; Russo, Mammarella, \& Avons, 2002). These results cannot be fully accounted for by encoding variability models (Melton, 1970), which are more relevant to free recall tasks than recognition or cued-recall tasks (Greene, 1989). They are also incompatible with one specific version of the deficient processing model which posits that the spaced items receive more voluntary rehearsal during repetition (Greene, 1989). Accordingly, Russo et al. (1998) proposed that the spacing effect in cued-memory tasks for unfamiliar stimuli is mediated by short-term perceptual priming. Specifically, stronger perceptual priming under the massed presentation condition leads to reduced perceptual processing of the second presentation and thus worse performance in the cued-recognition test that relies on the retrieval of structural-perceptual information of the targets. Consistent with this view, changing the color of the nonsense shapes (Cornoldi \& Longoni, 1977), the font of the nonwords (Mammarella et al., 2004; Russo et al., 2002), and the orientation of the faces (Mammarella et al., 2002) during massed presentation can all eliminate the spacing effect.

Wagner et al. (2000) proposed that SL reduces neural repetition suppression (RS), which results in enhanced encoding-related neural activity upon repeated presentations and better SM. This hypothesis is based on evidence from 
several lines of imaging research. First, studies have consistently found that stronger brain activity during encoding predicts better SM (e.g., Brewer, Zhao, Desmond, Glover, \& Gabrieli, 1998; Wagner et al., 1998). In particular, many studies found strong SM effects for faces in regions involved in visual processing such as the fusiform gyrus (Prince, Dennis, \& Cabeza, 2009; Golarai et al., 2007; Kirchhoff, Wagner, Maril, \& Stern, 2000; Wagner et al., 2000; Kuskowski \& Pardo, 1999). Second, manipulations on the depth of encoding (Otten, Henson, \& Rugg, 2001) and the familiarity of study material (Chee, Westphal, Goh, Graham, \& Song, 2003) that increase the neural activity during encoding can enhance SM. Third, the amplitude of RS at a longer lag is reduced compared with a shorter lag (Henson, Rylands, Ross, Vuilleumeir, \& Rugg, 2004).

To directly examine the hypothesis that lag time affects RS, Wagner et al. (2000) measured the BOLD response in a semantic judgment task (concrete/abstract) of three groups of words: one group that had been studied once 25 hours before the scan (long lag), one that had been studied immediately before the scan (short lag), and one that had not been studied before (once presented). Compared with the short-lag trials, long-lag trials showed better performance in a recognition memory test administered two days after the scan. The long-lag trials also showed less behavioral repetition priming and less neural RS in the left inferior frontal gyrus (LIFG) and the left fusiform gyrus. Moreover, participants who showed stronger neural RS in the LIFG had worse recognition memory, but only for the long-lag trials.

These seminal studies have triggered several intriguing questions that need to be further studied. First, the behavioral model proposed by Russo et al. (1998) was based on results from a cued-memory task. It is not clear whether such results can be replicated using the recognition memory task. Second, because the spaced items in the study of Wagner et al. (2000) were studied one day before, better memory could be achieved by memory consolidation. It thus remains to be seen whether a similar effect can be observed when spaced items and massed items are temporally adjacent. Third, neural repetition priming was measured by comparing the repeated items with once-presented items, which may or may not have been completely matched. Fourth, although later studies have shown that repetition priming and SM share common neural substrates (Turk-Browne, Yi, \& Chun, 2006; Kirchhoff et al., 2000), no study thus far has shown that neural RS, SM, and spacing share common neural substrates. Finally, in a behavioral study that aimed at replicating the study of Wagner et al., Stark, Gordon, and Stark (2008) failed to reveal any strong correlations between repetition priming and SM, either within or across subjects. An earlier study, however, found that stronger RS was associated with better recognition memory (Turk-Browne et al., 2006). These inconsistencies in research findings need to be addressed.

The present fMRI study tested the hypothesis that SL enhances SM by reducing RS during learning. Novel faces and a recognition memory task were used to specifically tap into effects of perceptual priming on memory performance. For the ML condition, four repetitions of a face were consecutively presented, whereas for the SL condition, each repetition was interleaved with a minimal interrepetition interval of six faces (maximum lag $=20$ items, mean lag $=12$ items) to reduce the perceptual priming effect (McKone, 1995). Massed trials and spaced trials were pseudorandomly mixed. A recognition memory test was conducted 1 hour after the scan. We predicted that if the spacing effect was mediated by RS, there should be overlapping neural substrates among SL, RS, and SM. Moreover, the level of RS during encoding should predict SM, that is, smaller RS would be associated with better memory performance. Finally, we predicted that SL should reduce RS and enhance SM.

\section{METHODS \\ Participants}

Twenty-four subjects ( 12 men, mean age $=22.8 \pm 2.91$ years old, ranging from 19 to 30 years) participated in this study. All subjects had normal or corrected-to-normal vision and were strongly right-handed as judged by Snyder and Harris's (1993) handedness inventory. None of them had a previous history of neurological or psychiatric disease. Informed written consent was obtained from the subjects before the experiment. This study was approved by the institution review board at Beijing Normal University.

\section{Materials}

Figure 1 illustrates the materials and experimental design. In total, 264 grayscale, unfamiliar, emotionally neutral faces were used in this study. Sixty faces were studied under the massed learning condition, and another 60 faces were studied under the SL condition, which were counterbalanced across participants. Another 120 faces were used as foils in the recollection memory tests. To minimize the primacy and recency effects, we added 24 faces ( 8 faces for each session, see below) at the beginning and the end of the study list. They were excluded from the behavioral and fMRI analyses. To prevent subjects from memorizing the faces through other features, we removed hair and ears from the pictures. All stimuli were normalized to the same size $(318 \times 340$ pixels $)$, spatial resolution, and brightness.

\section{fMRI Procedure and Task}

Subjects lay supine on the scanner bed and viewed visual stimuli back-projected onto a screen through a mirror attached onto the head coil. Foam pads were used to minimize head motion. Stimulus presentation and timing of all stimuli were achieved using E-prime (Psychology Software 
Figure 1. Experimental design. Each novel face was repeated four times, consecutively for massed learning (with jittered intertrial interval) but in an interleaved manner (with an interrepetition interval ranging from 6 to 20 trials) for spaced learning (SL). Each face was presented for $2 \mathrm{sec}$, followed by a blank lasting $2-5 \mathrm{sec}$ to improve the design efficiency. Four faces were added as fillers in the beginning and the end of the study list to eliminate the primacy and recency effects.

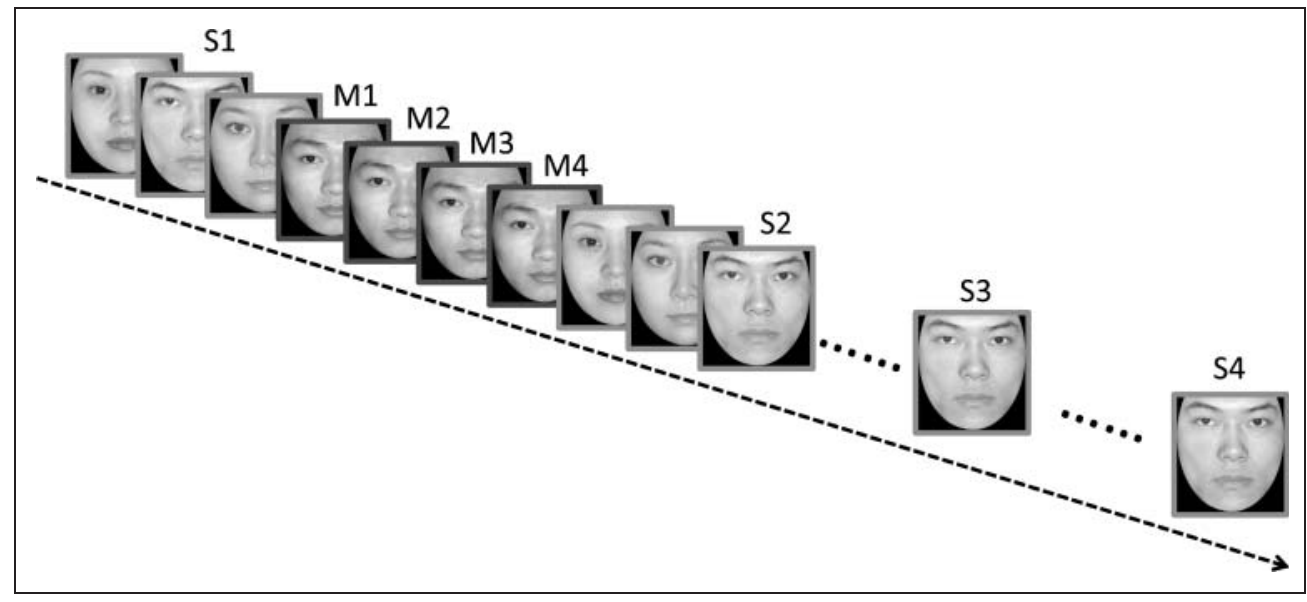

Tools, Inc., Pittsburgh, PA) on an IBM-compatible PC. During the scan, participants were explicitly instructed to intentionally memorize each face presented on the screen, and they were also told that a memory test would be conducted after the scanning session. Event-related design was used in this study, with SL and ML pseudorandomly mixed. Each face was repeated four times. For the ML condition, the four repetitions of a given face were grouped together with 0 interrepetition interval. In contrast, for the SL condition, the four repetitions were randomly spaced out, with an averaged interrepetition interval of 12, ranging from 6 to 20. For each trial, the stimulus was presented for $2 \mathrm{sec}$, followed by a random jitter (i.e., fixation) that lasted from 1 to $5 \mathrm{sec}$ (mean = $2.5 \mathrm{sec}$ ) to improve the design efficiency (Dale, 1999). To avoid the primacy and the recency effects, we placed four faces in the beginning and the end of the sequence, respectively. They were treated as fillers and encoded as nuisance variable in the fMRI data analysis. Participants finished three sessions of the memory task, each lasting $580 \mathrm{sec}$. In each session, 20 faces were studied under the SL condition and 20 were studied under the ML condition.

\section{Postscan Behavioral Test}

A recognition memory test was conducted to assess participants' memory performance 1 hour after the scan. During the recognition memory test, a total of 240 faces (half learned, half new) were randomly mixed together. For each stimulus, the subjects had to decide whether it had been learned before on a 6-point confidence scale, from 1 (definitely new) to 6 (definitely old). The stimulus remained on the screen until a response was made. The next item appeared after a 1-sec delay. There was no time pressure for the participants to finish the memory tests (Figure 2).

\section{MRI Data Acquisition}

Imaging data were acquired on a 3.0-T Siemens MRI scanner in the MRI Center at Beijing Normal University. Singleshot T2*-weighted gradient-echo, EPI sequence was used for functional imaging acquisition with the following parameters: repetition time/echo time $/ \theta=2000 \mathrm{msec} / 30 \mathrm{msec} /$ $90^{\circ}$, field of view $=200 \times 200 \mathrm{~mm}$, matrix $=64 \times 64$, and slice thickness $=4 \mathrm{~mm}$. Thirty contiguous axial slices parallel to AC-PC line were obtained to cover the whole
Figure 2. Behavioral effect of spaced learning. (A) Mean number of correct hits with high confidence ( $\geq 5$ on a 6-point scale, with $1=$ definitely new and $6=$ definitely old) for spaced (SL) and massed learning (ML) in recognition memory tests administered 1 hour after the scan. (B) Mean discriminability index $\left(d^{\prime}\right)$ for spaced and massed learning. (C) Mean lambda (bias) for spaced and massed learning. Error bars represent within-subject standard error.

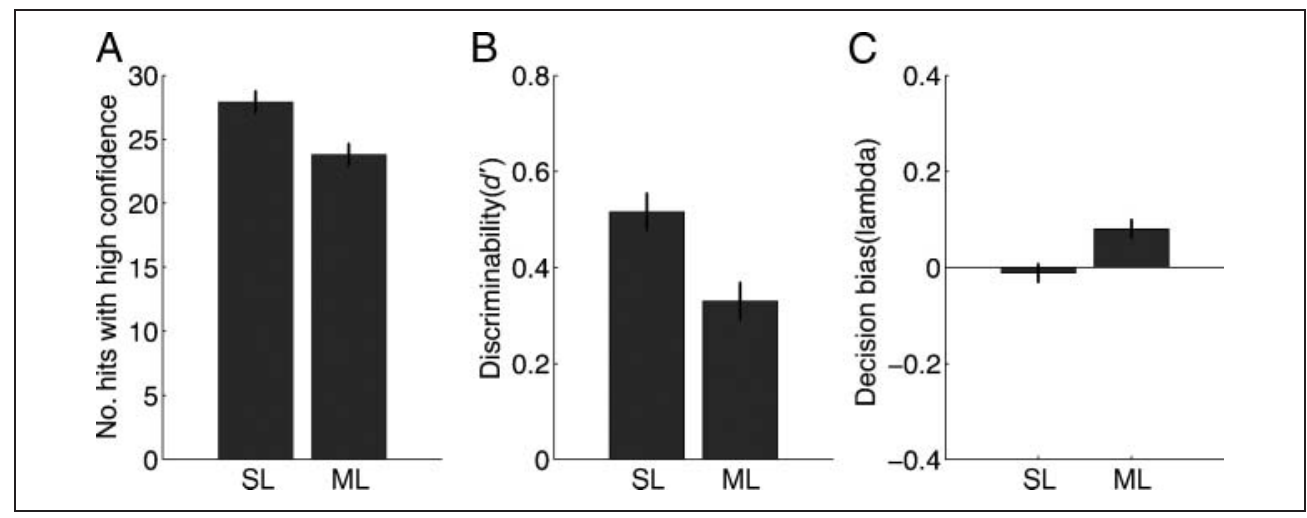


cerebrum and partial cerebellum. Anatomical MRI was acquired using a T1-weighted, three-dimensional, gradientecho pulse sequence. Parameters for this sequence were as follows: repetition time $/$ echo time $/ \theta=2530 \mathrm{msec} / 3.39 \mathrm{msec} /$ $7^{\circ}$, field of view $=256 \times 256 \mathrm{~mm}$, matrix $=192 \times 256$, and slice thickness $=1.33 \mathrm{~mm}$. One hundred twenty-eight (128) sagittal slices were acquired to provide a high-resolution structural image of the whole brain.

\section{Behavioral Data Analysis}

Two indices were used to describe the memory performance. The first index was simply the number of correct hits with high confidence (rated as 5 and 6). Because this index was biased by individuals' response criteria, we calculated a second index. Afterward (Snodgrass \& Corwin, 1988), we computed the discriminability index (i.e., $d^{\prime}$ ) using the following formula: $d^{\prime}=Z_{\text {(hit rate) }}-Z_{\text {(false alarm) }}$. The lambda (bias) score was calculated using the following formula: lambda $=-1 / 2\left(Z_{\text {(hit rate) }}+Z_{\text {(false alarm) }}\right)$. For all indices, paired $t$ test was conducted to examine the effect of learning condition (SL vs. ML).

\section{Image Preprocessing and Statistical Analysis}

Image preprocessing and statistical analysis were carried out using FEAT (fMRI Expert Analysis Tool) version 5.98, part of the FSL (FMRIB software library, version 4.1, www. fmrib.ox.ac.uk/fsl). The first three volumes before the task were automatically discarded by the scanner to allow for T1 equilibrium. The remaining images were then realigned to correct for head movements (Jenkinson \& Smith, 2001). Translational movement parameters never exceeded 1 voxel in any direction for any subject or session. Data were spatially smoothed using a 5-mm FWHM Gaussian kernel. The data were filtered in the temporal domain using a nonlinear high-pass filter with a 60-sec cutoff. A two-step registration procedure was used whereby EPI images were first registered to the MPRAGE structural image and then into the standard Montreal Neurological Institute (MNI) space using affine transformations (Jenkinson \& Smith, 2001). Registration from MPRAGE structural image to the standard space was further refined using FNIRT nonlinear registration (Andersson, Jenkinson, \& Smith, 2007a, 2007b). Statistical analyses were performed in the native image space, with the statistical maps normalized to the standard space prior to higher level analysis.

Two general linear models within the FILM module of FSL were used to model the data. In the first model that examined the relations among SL, repetition priming, and $\mathrm{SM}$, the faces were separately modeled according to their SM (remembered vs. forgotten) for each learning condition (massed vs. spaced) and each repetition (Repetitions 1 to 4 ). Only the old faces that were recognized with high confidence score (5 and 6) were considered as remembered items (Otten et al., 2001). Similarly, only the old faces that were judged as new with high confidence score (1 and
2) were considered as forgotten items. Items with a score of 3 and 4 (i.e., low confidence) were encoded as one nuisance variable. The first and the last four filler faces were also encoded as a separate nuisance variable. Null events were not explicitly modeled and therefore constituted an implicit baseline. For each subject, seven contrast images were computed, including the main effects of SL (spaced - massed), RS (first repetition - second to fourth repetitions), and SM effect (remembered - forgotten) and their two-way and three-way interactions. A higher level analysis created cross-run contrasts for each subject for a set of contrast images using a fixed effect model. These were then input into a random-effect model for group analysis, using FLAME (FMRIB's Local Analysis of Mixed Effects) Stage 1 only with automatic outlier detection (Woolrich, 2008; Woolrich, Behrens, Beckmann, Jenkinson, \& Smith, 2004; Beckmann, Jenkinson, \& Smith, 2003).

The second model examined individual differences in encoding-related brain activation and their relations to memory performance. Because the number of remembered faces did not necessarily equal to that of forgotten faces for each subject, the sum of the activation for remembered and forgotten faces from the first model may not correctly reflect the overall encoding-related activation. As a result, in the second model, all faces, regardless of being remembered or forgotten in the recognition test, were encoded as one variable, separately for each repetition and each learning condition. Two contrasts, the overall activation (summed across four repetitions) and the amplitude of neural RS, were separately computed for each learning condition (massed and spaced). At the higher level analysis, individuals' discriminability index $\left(d^{\prime}\right)$ was added as a covariate to examine the relations between individuals' memory performance and encoding-related brain activities.

For both models, unless otherwise noted, group images were thresholded using cluster detection statistics, with a height threshold of $Z>2.3$ and a cluster probability of $p<.05$, corrected for whole-brain multiple comparisons using Gaussian random field theory.

\section{Conjunction Analysis}

If SL indeed could reduce RS in regions showing the SM effect, SL, RS, and SM should share overlapping neural substrates. This was tested with a conjunction analysis using the procedure suggested by Nichols, Brett, Andersson, Wager, and Poline (2005). Group maps for each contrast were thresholded individually at $Z=2.3$ (corrected for multiple comparisons at the whole-brain level), binarized, and multiplied, which resulted in brain regions shared by SL, RS, and SM.

\section{ROI Analyses}

ROI analyses were conducted to examine the interactions among repetition priming, $\mathrm{SL}$, and $\mathrm{SM}$ in the brain regions 
showing a significant SM effect. Because there was no significant interaction between learning condition and SM effect (see Results), voxels in the bilateral fusiform gyrus that showed significant SM effects were defined as ROIs. ROI analyses were performed by extracting parameter estimates (betas) of each event type from the fitted model and averaging across all voxels in the cluster for each subject. Percent signal changes were calculated using the following formula: [contrast image / (mean of run)] $\times$ ppheight $\times$ $100 \%$, where ppheight is the peak height of the hemodynamic response versus the baseline level of activity (Mumford, 2007).

To make sure that the cross-subject brain-behavior correlation was not driven by outlier(s), we performed another ROI analysis on nonindependently defined ROIs. To do so, we defined the left fusiform that showed the most significant correlation with memory performance as ROI by growing a 6-mm diameter sphere around the local maxima. Scatterplots were made to show the correlation between brain activation and memory performance.

\section{RESULTS}

\section{Behavioral Data: SL Enhanced SM}

First, we examined whether SL resulted in any behavioral advantages in recognition memory. Consistent with previous observations, SL was associated with more hits with high confidence than was massed learning. The numbers of hits were 27.9 for SL and 23.8 for $\mathrm{ML}, t(23)=3.52, p=.0018$; the $d^{\prime}$ was .52 and .33 for SL and ML, respectively, $t(23)=$ $3.49, p=.002$. On average, the bias score (lambda) was not significantly different from 0 in both conditions $(-.01$ and $.08, p=.81$ and $p=.10$, respectively), although subjects somewhat more likely to say "yes" for the spaced items than for the massed items $(t=3.46, p=.002)$. The mean false alarm rate was $40 \%$. After excluding eight subjects whose $d^{\prime}$ score was lower than .15, we still found a significant spacing effect; $d^{\prime}: t(15)=2.95, p=.01$; hits: $t(15)=3.58, p=.003$. Because $d^{\prime}$ is unaffected by individuals' decision criterion, it was then used as the behavioral index in our analysis of neural activities during memory encoding.

\section{fMRI Results}

Having shown the behavioral effect of SL, we then examined the hypothesis that SL enhanced SM by reducing RS in the brain regions that support SM. If this hypothesis is true, we should expect that (1) these brain regions exhibited common SM effects for spaced and massed learning conditions, (2) the same regions showing the SM effects also showed RS effects and were affected by SL, (3) subsequently remembered items showed smaller RS, and (4) SL significantly reduced RS. Our data supported all these hypotheses.

\section{Common SM Mechanisms for SL and ML}

To examine the first hypothesis, we first identified the brain regions that showed SL effects. A comparison of remembered items (high-confidence hits) and forgotten items revealed significantly stronger activation in the left ( $x y z$ in MNI: $-42,-58,-16, Z=4.5$ ) and right (MNI: 36, $-68,-18, Z=4.8)$ fusiform cortices that extended to the inferior occipital cortex as well as the right angular gyrus (Figure 3C, Table S1). No significant interaction between learning condition and the SM effect was found in this area, suggesting that bilateral fusiform cortex is important for SM under both spaced and massed learning conditions.

\section{Common Neural Mechanisms for SM, RS, and SL}

We then examined whether the same regions that showed SM effects were also affected by SL and RS. SL and ML differed significantly in neural activations in the left (MNI: $-44,-62,-24, Z=3.71$ ) and right (MNI: 48, $-62,-8$, $Z=4.0$ ) fusiform cortices (Figure 3A, Table S2). Similarly, by comparing the first repetition with the subsequent repetitions, we found strong RS effects in the left (MNI: -44 , $-66,-8, Z=4.19$ ) and right (MNI: 42, $-58,-20, Z=$ 3.70) fusiform cortices (Figure 3C, Table S3). As we hypothesized, conjunction analysis revealed that SL, repetition priming, and SM shared common neural mechanisms in bilateral fusiform cortex (Figure 3D, Table S4).

\section{SL Enhanced SM by Reducing Repetition Suppression}

In a subsequent analysis to examine the interactions among SM, RS, and SL, we extracted the BOLD signal changes for each condition in bilateral fusiform cortex that showed an SM effect and subjected them to a three-way repeated measures ANOVA (Figure 3E). This analysis revealed two significant interactions. First, RS was significantly smaller for remembered faces than for forgotten faces, $F(1,23)=$ $5.07, p=.034$, indicating a significant association between RS and recognition memory. Second, RS was significantly smaller for SL than for ML, $F(1,23)=4.66, p=.042$, suggesting that SL reduced RS. There was no significant interaction between learning condition and the SM effect, $F(1,23)=0.076, p=.78$, nor was there a threeway interaction, $F(1,23)=0.006$, ns. Taken together, these results provide direct evidence that SL reduced RS in the brain regions that were responsible for memory encoding, which in turn led to increased activation and better SM.

\section{Summed Activity, But Not RS, Predicted Individuals' Memory Performance}

The above analyses suggested that for learning involving repeated practices, the summed activation across repetitions predicted SM within subjects. As smaller RS during 
Figure 3. fMRI results. Neural effects of (A) spaced learning (SL), (B) repetition suppression (RS), (C) subsequent memory (SM), and (D) their conjunction thresholded at $Z>2.3$ (whole-brain corrected) are rendered onto a population-averaged surface atlas using multifiducial mapping (Van Essen, 2005). (E) Plot of percent signal changes in regions showing a significant SL effect. Error bars represent within-subject standard error. $\mathrm{M}=$ massed condition; $\mathrm{S}=$ spaced condition; 1 = first presentation; $2-4=$ second to fourth presentation.

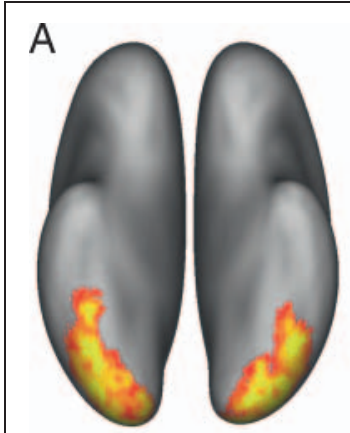

Spaced learning effect (Spaced > Massed)

D

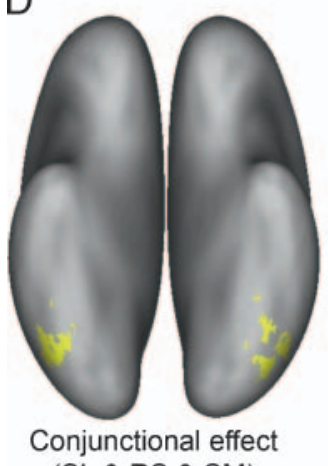

(SL \& RS \& SM)
B

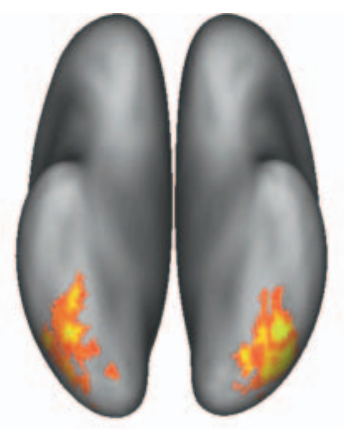

Repetition Suppression (First > Last repetition)
Subsequent memory effect (Remembered > Forgotten)
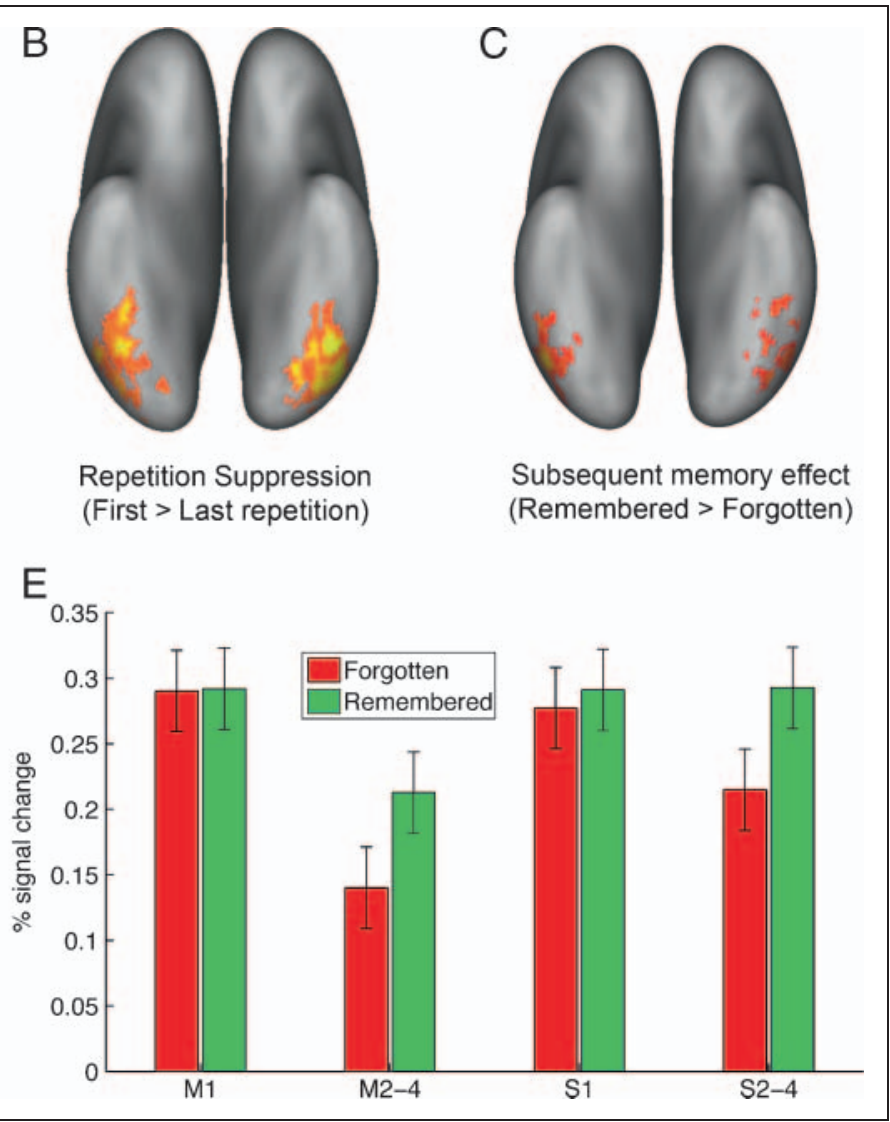

learning resulted in stronger summed activation, we also found that smaller RS also predicted better SM within subjects. To further examine whether they could also predict individuals' memory performance, we did a whole-brain correlation analysis between each individual's memory performance $\left(d^{\prime}\right)$ and the two neural indices: summed activity and RS. This cross-subject analysis revealed that only the summed activity in the bilateral fusiform cortex, which extended to the right occipital cortex, was significantly correlated with individuals' memory performance for both SL and massed learning (Figure 4, Table S5). The same results were also obtained when only subjects with abovechance recognition memory $\left(d^{\prime}>0.15\right)$ were included (massed: $r=.82, p<.001$; spaced: $r=.75, p<.001$ ). No significant correlations were found between RS and memory performance.

It should be noted that the summed brain activity (R_sum) across repetitions is determined by the neural response to the first presentation (R1) and the amplitude of RS, as expressed in the following formula: $\mathrm{R} \_$sum $=\mathrm{R} 1 \times$ rep $\mathrm{RS} \times($ rep -1$)$, where rep represents the number of repetitions (i.e., four in the present study), and the RS was calculated by subtracting the mean activity for Repetitions 2 to 4 from the first repetition. We then further correlated the R1 with individuals' memory performance. It turned out that R1 itself could effectively predict SM under both massed $(r=.71, p<.001)$ and spaced conditions $(r=$ $.70, p<.001)$. Furthermore, we found that R1 accounted for most of the variance of the summed activity (massed: $r=.965, p<.001$; spaced: $r=.995, p<.001$ ) and also was correlated with RS (massed: $r=.46, p=.025$; spaced: $r=.37, p=.077$ ). Because individuals' responses to the first presentation were closely correlated with their memory performance, little variance of the latter remained to be explained by other variables such as RS. This result suggests that cross-subject analysis is unlikely to yield a significant correlation between RS and $d^{\prime}$. We will return to this point in the Discussion.

\section{DISCUSSION}

By repeating stimuli according to different schedules (massed vs. spaced) and by using an SM approach, the present fMRI study was able to examine the relations among SL, RS, and SM. We found that remembering was associated with smaller RS during learning, and SL reduced repetition priming and enhanced recognition memory. These results provide a neural account for the spacing effect in learning, which seems to involve the interaction between neural RS and episodic memory.

Consistent with previous neural studies (Callan \& Schweighofer, 2009; Wagner et al., 2000), we found that spacing can affect both neural RS and episodic memory, suggesting that they shared common processing factors. In addition to spacing, it has been shown that neural RS 
and episodic memory can be simultaneously affected by attention (Turk-Browne et al., 2006). Extending previous results of overlapping neural substrates for neural RS and recognition memory (Turk-Browne et al., 2006; Kirchhoff et al., 2000), the conjunction analysis in the present study revealed overlapping neural substrates for spacing, RS, and recognition memory. The shared neural substrates provide a potential neural basis for SL to modulate neural RS and also to affect recognition memory.

Our study revealed three additional important results about the neural mechanisms underlying the spacing effect. First, we found that both within and across subjects, the summed activity pooled across repetitions during memory encoding could predict SM. This finding extends previous studies examining the SM effect involving single learning event (e.g., Brewer et al., 1998; Wagner et al., 1998). Second, we found that within subjects, items with less RS were associated with better recognition memory, providing direct evidence that neural RS impairs recognition memory. Finally, we found that SL could significantly reduce $\mathrm{RS}$ in the brain regions that support episodic memory encoding. Taken together, these results suggest that one important neural mechanism for the spacing effect is the reduced neural RS and the enhanced encodingrelated brain activities. Our study, together with other imaging studies on SL (Callan \& Schweighofer, 2009; Wagner et al., 2000), suggests that deficient processing during memory encoding, as reflected by the weaker brain activation as a result of RS, hinders long-term cognition memory.

The imaging results are in agreement with the deficient processing hypothesis in general and the short-term priming hypothesis in particular, which posits that repetition priming reduces the processing of subsequently presented items, thus reduces the overall strength of encoding processes. Because of the use of novel faces as stimuli and a recognition task, our data cannot be accounted for by the encoding variability hypothesis or the active rehearsal hypothesis (Callan \& Schweighofer, 2009; Wagner et al., 2000). One factor that contributes to deficit processing is attention because subjects might pay less attention to the recently studied and familiar stimuli. Subjects could also reduce their attention because of the predictability of the stimuli, which could account for part of the neural suppression in the third and fourth repetition of the massed learning condition (but not that from the first to second presentations and that in the spaced condition). In the present study, we used an explicit memory-encoding task to minimize the attention effect. Results showed that even under our intentional memory condition, massed presentations still led to deficient processing, echoing the idea that the spacing effect is not under voluntary control (Hintzman, 1974). One limitation of this paradigm,
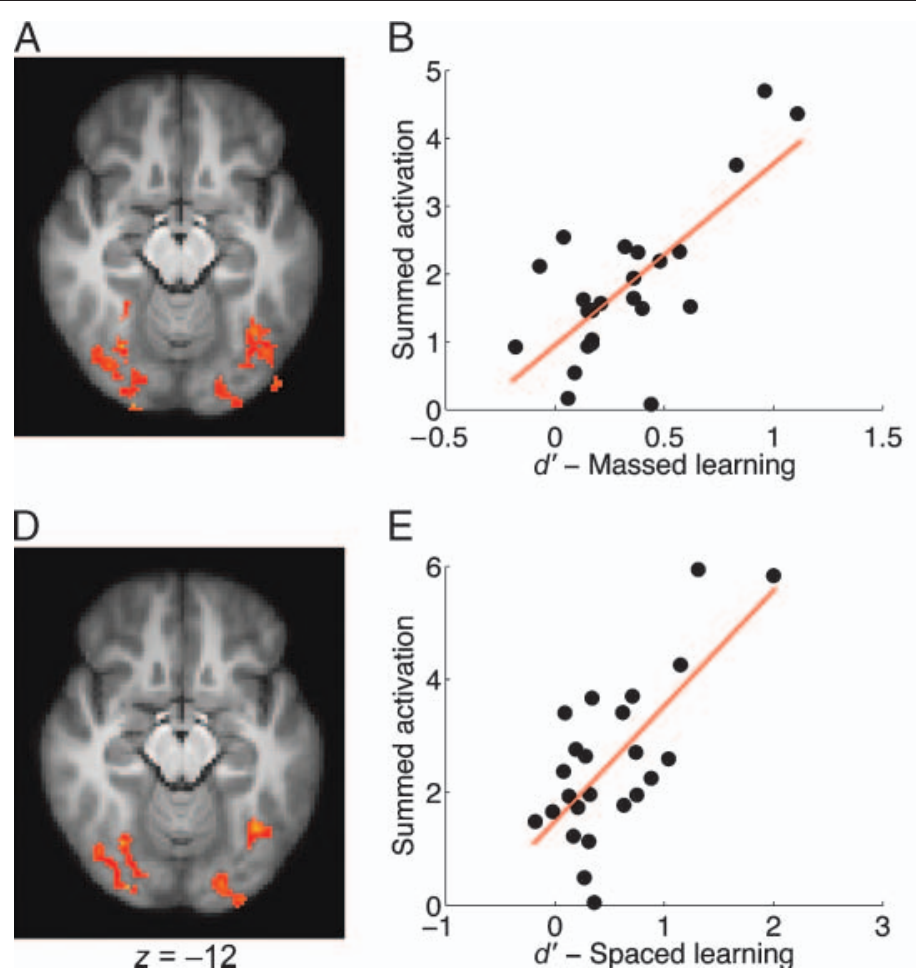
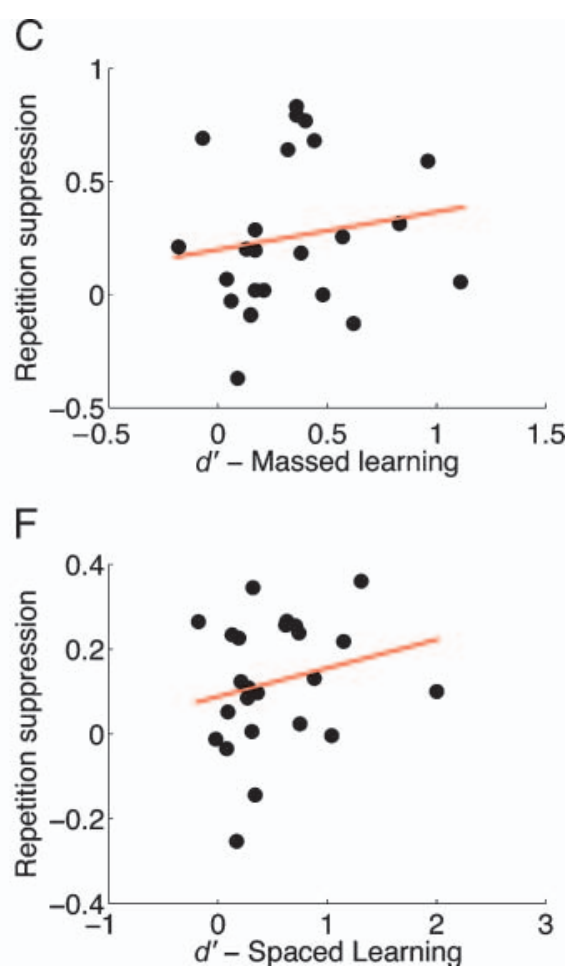

Figure 4. Summed activity predicted individuals' memory performance. The bilateral fusiform showed significant correlations $(Z>2.3$, whole-brain corrected) between summed activity and subsequent memory (SM; $d^{\prime}$ ) for both massed learning (A) and spaced learning (SL; D). (B and E) The scatterplots for massed and SL, respectively. No significant correlation was found between repetition suppression (RS) and memory performance for either massed (C) or SL (F). Please note that the ROI was not independently defined, and the absolute correlation coefficients should be treated cautiously. 
however, is that it did not provide a behavioral index of repetition priming. Although many studies have found strong and causal relations between neural RS and behavioral repetition priming (Wig, Grafton, Demos, \& Kelley, 2005; Maccotta \& Buckner, 2004), future studies need to directly measure behavioral repetition priming and neural RS and examine their relations with recognition memory.

It is interesting to note that, in comparing neural activities between the spaced and massed learning conditions, we found that the mean level of neural activity associated with the remembered items in the ML condition was the same as that for the forgotten items in the SL condition, $t(23)=0.09, p=.92$. Similarly, levels of neural suppression did not differ between the remembered items in the ML condition and the forgotten items in the SL condition, $t(23)=0.39, p=.69$. These results seem to suggest that although SL can effectively enhance neural activity and improve memory, massed learning may be neurally more efficient in memory encoding. That is, compared with the spaced condition, a weaker input from the visual system would be sufficient to register memory under the massed condition because of the residual neuronal responses. Under the ML condition, the brain activation that is not critical for memory encoding can be effectively eliminated through RS, although they would have been present during visual object processing. Future studies need to examine these speculations.

A more general question for future studies is the possible interaction between repetition priming and recognition memory. Both behavioral and neural models seem to suggest that the spacing effect is mediated by the interaction between repetition priming and recognition memory (Wagner et al., 2000), although they are believed to reflect two dissociable memory mechanisms, such as explicit versus implicit memory (Tulving \& Schacter, 1990) or declarative versus nondeclarative memory (Squire, 1992). Cumulative evidence from both behavioral and functional imaging studies suggests that repetition priming and recognition memory may not be completely independent. For example, the same brain lesions can lead to impairment in both explicit and implicit memory (Jernigan \& Ostergaard, 1993), although the typically large lesions in these patients make it difficult to exclude the possibility that these behavioral impairments are caused by lesions in different subregions. In addition, it has been argued on the basis of computational modeling (e.g., Kinder \& Shanks, 2003) that dissociations between implicit and explicit memory measures do not necessarily reflect multiple underlying systems. Moreover, the same LIFG region that often shows repetition priming effects is also thought to play a role in explicit memory encoding and retrieval (Schott et al., 2005; Lustig \& Buckner, 2004; Kirchhoff et al., 2000; also see Wagner et al., 1998, 2000). The present study also found that the same bilateral fusiform region is involved in both repetition priming and explicit memory encoding.
Although the overlapping neural substrates provide a potential neural basis for the two memory forms to interact, quantitative examinations of the relations among repetition priming, neural RS, and recognition memory has thus far produced mixed results. For example, although several studies have found that SL could simultaneously reduce repetition priming and enhance memory, only the present study revealed that within subjects, stronger RS was associated with worse recognition memory (see a different finding in Turk-Browne et al., 2006). In contrast, Wagner et al. (2000) found negative cross-subjects correlations between repetition priming and recognition memory performance and only under the SL condition.

Results from the present study provide a way to potentially reconcile these mixed results and also provide useful guidance for future studies. Consistent with previous studies using one-shot learning, we found that the summed brain activity (R_sum) across repetitions was a good predictor of SM. R_sum was determined by the brain response to the first presentation (R1) and the amplitude of RS. More importantly, R1 was also significantly correlated with RS and accounted for most of the variance of the summed activation. This finding may explain why very few studies found significant correlations between RS and recognition memory across subjects. It may also explain the positive interaction found by Turk-Browne et al. (2006). In that study, activation was significantly stronger for the remembered items than for the forgotten items during the first presentation. Considering that stronger R1 is usually associated with larger RS when no manipulation of repetition lag and RS is used, it is not surprising that the remembered items were associated with stronger RS in their study. These results suggest that for future studies to observe a significant correlation between RS and SM, it would be necessary to reduce the variance of $\mathrm{R} 1$ and to increase the variance of RS, both within and across subjects. On potential fruitful approach is to study this correlation within subjects (1) by carefully reducing the variance in material, which could affect the brain responses to the first presentation, and (2) by manipulating the repetition lag to change RS. Significant cross-subjects correlations are more difficult to obtain because of the difficulties involved in controlling the variance across subjects and manipulating their RS.

Taken together, our results demonstrate that SL enhances recognition memory by reducing RS, which provides a neural account of the spacing effect and suggests that the spacing effect might entail the interaction between neural RS and explicit memory. More studies are required to examine the possible interactions among repetition priming, neural RS, and explicit memory.

\section{Acknowledgments}

The authors thank Professor Robert Bjork for his helpful comments. This study was supported by the Program for New Century Excellent Talents in University, the National Science Foundation 
(grants BCS 0823624 and BCS 0823495), the National Institute of Health (grant HD057884-01A2), and the 111 Project of China (grant B07008).

Reprint requests should be sent to Gui Xue, Department of Psychology, University of Southern California, Los Angeles, CA 90089, or via e-mail: guixue@gmail.com or Qi Dong, Institute of Cognitive Neuroscience and Learning, Beijing Normal University, 100875 Beijing, China, or via e-mail: dongqi@bnu.edu.cn.

\section{REFERENCES}

Andersson, J., Jenkinson, M., \& Smith, S. (2007a). Non-linear optimisation (FMRIB Tech. Rep. No. TR07JA1). Retrieved from www.fmrib.ox.ac.uk/analysis/techrep.

Andersson, J., Jenkinson, M., \& Smith, S. (2007b). Non-linear registration, aka spatial normalisation (FMRIB Tech. Rep. No. TR07JA2). Retrieved from www.fmrib.ox.ac.uk/ analysis/techrep.

Arthur, W., Bennett, W., Stanush, P., \& McNelly, T. (1998). Factors that influence skill decay and retention: A quantitative review and analysis. Human Performance, 11, 57-101.

Beckmann, C. F., Jenkinson, M., \& Smith, S. M. (2003). General multilevel linear modeling for group analysis in fMRI. Neuroimage, 20, 1052-1063.

Brewer, J., Zhao, Z., Desmond, J., Glover, G., \& Gabrieli, J. (1998). Making memories: Brain activity that predicts how well visual experience will be remembered. Science, 281, 1185.

Callan, D. E., \& Schweighofer, N. (2009). Neural correlates of the spacing effect in explicit verbal semantic encoding support the deficient-processing theory. Human Brain Mapping, 31, 645-659.

Cepeda, N., Pashler, H., Vul, E., Wixted, J., \& Rohrer, D. (2006). Distributed practice in verbal recall tasks: A review and quantitative synthesis. Psychological Bulletin, 132, 354

Chee, M., Westphal, C., Goh, J., Graham, S., \& Song, A. (2003). Word frequency and subsequent memory effects studied using event-related fMRI. Neuroimage, 20, 1042-1051.

Cornoldi, C., \& Longoni, A. (1977). The MP-DP effect and the influence of distinct repetitions on recognition of random shapes. Italian Journal of Psychology, 4, 65-76.

Dale, A. M. (1999). Optimal experimental design for event-related fMRI. Human Brain Mapping, 8, 109-114.

Donovan, J., \& Radosevich, D. (1999). A meta-analytic review of the distribution of practice effect: Now you see it, now you don't. Journal of Applied Psychology, 84, 795-805.

Ebbinghaus, H. (1964). Memory: A contribution to experimental psychology (H. R. C. Bussenius, Trans.). New York: Dover Publications. (Original work published 1885).

Golarai, G., Ghahremani, D., Whitfield-Gabrieli, S., Reiss, A., Eberhardt, J., Gabrieli, J., et al. (2007). Differential development of high-level visual cortex correlates with category-specific recognition memory. Nature Neuroscience, $10,512-522$.

Greene, R. (1989). Spacing effects in memory: Evidence for a two-process account. Learning and Memory, 15, 371-377.

Henson, R. N., Rylands, A., Ross, E., Vuilleumeir, P., \& Rugg, M. D. (2004). The effect of repetition lag on electrophysiological and haemodynamic correlates of visual object priming. Neuroimage, 21, 1674-1689.

Hintzman, D. (1974). Theoretical implications of the spacing effect. In R. Solso (Ed.), Theories in cognitive psychology: The Loyola symposium (pp. 77-99). Potomac, MD: Erlbaum.
Jenkinson, M., \& Smith, S. (2001). A global optimisation method for robust affine registration of brain images. Medical Image Analysis, 5, 143-156.

Jernigan, T., \& Ostergaard, A. (1993). Word priming and recognition memory are both affected by mesial temporal lobe damage. Neuropsychology, 7, 14-26.

Kinder, A., \& Shanks, D. (2003). Neuropsychological dissociations between priming and recognition: A single-system connectionist account. Psychological Review, 110, 728-743.

Kirchhoff, B., Wagner, A., Maril, A., \& Stern, C. (2000). Prefrontal-temporal circuitry for episodic encoding and subsequent memory. Journal of Neuroscience, 20, 6173.

Kuskowski, M., \& Pardo, J. (1999). The role of the fusiform gyrus in successful encoding of face stimuli* 1. Neuroimage, 9, 599-610.

Lustig, C., \& Buckner, R. (2004). Preserved neural correlates of priming in old age and dementia. Neuron, 42, 865-875.

Maccotta, L., \& Buckner, R. (2004). Evidence for neural effects of repetition that directly correlate with behavioral priming. Journal of Cognitive Neuroscience, 16, 1625-1632.

Mammarella, N., Avons, S., \& Russo, R. (2004). A short-term perceptual priming account of spacing effects in explicit cued-memory tasks for unfamiliar stimuli. European Journal of Cognitive Psychology, 16, 387-402.

Mammarella, N., Russo, R., \& Avons, S. (2002). Spacing effects in cued-memory tasks for unfamiliar faces and nonwords. Memory \& Cognition, 30, 1238-1251.

McKone, E. (1995). Short-term implicit memory for words and nonwords. Journal of Experimental Psychology: Learning, Memory, and Cognition, 21, 1108-1125.

Melton, A. (1970). The situation with respect to the spacing of repetitions and memory. Journal of Verbal Learning and Verbal Behavior, 9, 596-606.

Mumford, J. (2007). A guide to calculating percent change with featquery (Unpublished Tech. Rep.). Retrieved from http://mumford.bol.ucla.edu/perchange_guide.pdf.

Nichols, T., Brett, M., Andersson, J., Wager, T., \& Poline, J. (2005). Valid conjunction inference with the minimum statistic. Neuroimage, 25, 653-660.

Otten, L., Henson, R., \& Rugg, M. (2001). Depth of processing effects on neural correlates of memory encoding: Relationship between findings from across- and within-task comparisons. Brain, 124, 399.

Prince, S., Dennis, N., \& Cabeza, R. (2009). Encoding and retrieving faces and places: Distinguishing process- and stimulus-specific differences in brain activity. Neuropsychologia, 47, 2282-2289.

Russo, R., Mammarella, N., \& Avons, S. (2002). Toward a unified account of spacing effects in explicit cued-memory tasks. Journal of Experimental Psychology: Learning, Memory, and Cognition, 28, 819-829.

Russo, R., Parkin, A., Taylor, S., \& Wilks, J. (1998). Revising current two-process accounts of spacing effects in memory. Journal of Experimental Psychology: Learning, Memory, and Cognition, 24, 161-172.

Schott, B., Henson, R., Richardson-Klavehn, A., Becker, C., Thoma, V., Heinze, H., et al. (2005). Redefining implicit and explicit memory: The functional neuroanatomy of priming, remembering, and control of retrieval. Proceedings of the National Academy of Sciences, U.S.A., 102, 1257-1262.

Snodgrass, J., \& Corwin, J. (1988). Pragmatics of measuring recognition memory: Applications to dementia and amnesia. Journal of Experimental Psychology: General, 117, 34.

Snyder, P., \& Harris, L. (1993). Handedness, sex, and familial sinistrality effects on spatial tasks. Cortex, 29, 115. 
Squire, L. (1992). Declarative and nondeclarative memory: Multiple brain systems supporting learning and memory. Journal of Cognitive Neuroscience, 4, 232-243.

Stark, S., Gordon, B., \& Stark, C. (2008). Does the presence of priming hinder subsequent recognition or recall performance? Memory, 16, 157-173.

Tulving, E., \& Schacter, D. (1990). Priming and human memory systems. Science, 247, 301.

Turk-Browne, N. B., Yi, D.-J., \& Chun, M. M. (2006). Linking implicit and explicit memory: Common encoding factors and shared representations. Neuron, 49, 917-927.

Van Essen, D. (2005). A population-average, landmark- and surface-based (PALS) atlas of human cerebral cortex. Neuroimage, 28, 635-662.

Wagner, A. D., Maril, A., \& Schacter, D. L. (2000). Interactions between forms of memory: When priming hinders new episodic learning. Journal of Cognitive Neuroscience, 12(Suppl. 2), 52-60.

Wagner, A. D., Schacter, D., Rotte, M., Koutstaal, W., Maril, A., Dale, A., et al. (1998). Building memories: Remembering and forgetting of verbal experiences as predicted by brain activity. Science, 281, 1188

Wig, G., Grafton, S., Demos, K., \& Kelley, W. (2005).

Reductions in neural activity underlie behavioral components of repetition priming. Nature Neuroscience, $8,1228-1233$.

Woolrich, M. (2008). Robust group analysis using outlier inference. Neuroimage, 41, 286-301.

Woolrich, M. W., Behrens, T. E., Beckmann, C. F., Jenkinson, M., \& Smith, S. M. (2004). Multilevel linear modelling for fMRI group analysis using Bayesian inference. Neuroimage, $21,1732-1747$. 